\title{
DEPENDABLE ONLINE APPOINTMENT BOOKING SYSTEM FOR NHIS OUTPATIENT IN NIGERIAN TEACHING Hospitals
}

\author{
Adebayo Peter Idowu ${ }^{1}$, Olajide Olusegun Adeosun $^{2}$, and Kehinde Oladipo Williams ${ }^{3}$ \\ ${ }^{1}$ Department of Computer Science \& Engineering, Obafemi Awolowo University, Ile-Ife, Nigeria \\ ${ }^{2}$ Computer Science \& Engineering Dept., Ladoke Akintola University of Technology, Ogbomoso, \\ Nigeria \\ ${ }^{3}$ Department of Phyiscal and Computer Sciences, College of Natural and Applied Sciences, \\ McPherson University, Ajebo, Ogun State, Nigeria.
}

\begin{abstract}
The number of missed appointments in healthcare institutions in Nigeria caused problems, hence the need for integrated healthcare system to intervene and provide seamless care for patients. Appointment scheduling system lies at the intersection of providing efficiency and timely access to health services. This research presents an online National Health Insurance Scheme (NHIS) Outpatient Medical Appointment Booking System where NHIS patients can access and view any available personnel or doctor schedule in order to book an appointment with the corresponding time as specified by the available doctor. The system was developed using PhP, macromedia dreamweaver, apache and MYSQL. This is to ensure that the application is robust, cheap and is able to run on different platforms. The system provides the platform to facilitate the booking and management of patients' appointment bookings. Patients can also view their appointment reports. It also provides the healthcare workers an easy access to manage patients' appointments and to generate relevant reports.
\end{abstract}

\section{KEYWORDS}

NHIS, Medical Appointment, Booking system, Healthcare System.

\section{INTRODUCTION}

In this fast driven society, where the current climate in the healthcare sector demands efficiency and patients' satisfaction in medical care delivery. The numbers of missed appointments, unnecessary waste of patient's appointment time have caused an impending problem for healthcare institutions (Chua, 2010). Hence, there is need for integrated healthcare system to intervene and provide seamless care for both inpatients and outpatients. Therefore, an appointment booking system lies at the intersection of providing efficient and timely access to health services. Outpatient appointment booking is a subject of great interest to hospitals and other medical institutions (Koole, 2007).

The research on appointment scheduling has a long history, starting with the work of Welch and Bailey (1952). Their most famous result is the so-called Bailey-Welch appointment schedule, which states that two patients should be planned at the start of the day, and the other patients evenly spaced throughout the day, to offset the bad effects of no-shows and patient lateness 
(Welch and Bailey, 1952). Bailey (1952) already announced that an appointment system is a trade-off between doctors' and patients' waiting times. Although outpatient clinic's average internal waiting times are long, doctors frequently have idle time. Patients who do not show up or who are late for their appointments cause idle time for doctors, leading to temporary underutilization of the outpatient clinic's capacity. Gaps in the appointment schedules also cause underutilization of the doctor's time (Bailey, 1954).

Research on outpatient clinics shows that waiting times are patients' main dissatisfaction with hospital services (Huang, 1994). According to doctors and personnel, overtime and peak workloads are potential threats for the quality of care and the quality of labor, because they increase stress and time pressure. This research focuses on outpatient scheduling as a means to solve these problems for outpatient clinics. According to Cayirli (2003), access time is the time between the patient's request for an appointment and his arrival at the outpatient clinic. A patient's internal waiting time is the period between the scheduled starting time and the actual starting time of his consultation. Waiting time due to a patient's early arrival is extracted from the internal waiting time, since it is not a consequence of the appointment system (Veral, 2003).

Accessibility to healthcare at affordable cost constitutes a high challenge in Nigerian like most African countries. The provision of quality, accessible and affordable healthcare remains a serious problem (Oba, 2008) and due to this fact, Nigerian was ranked to be low in healthcare delivery by international organisations. In other to address the situation in the health sector, and to provide universal access to quality healthcare service in the country, National Health Insurance Scheme (NHIS) was introduced in Nigeria (Johnson, 2009); with the aim of providing universal access to quality healthcare to all Nigerians. In 1993, the Federal Government directed the Federal Ministry of Health to start the scheme in the country in 1999 and the scheme was modified to cover more people (Adesina, 2009). NHIS became operational after it was officially launched by the Federal Government in 2005 (Kannegiesser, 2009).

The purpose of NHIS is to ensure the provision of health insurance "which shall entitle insured persons and their dependents the benefit of prescribed good quality and cost effective health services" (NHIS, 1999). Because of the cost effective of the scheme, there are more beneficiary that are entitle to the medical benefit and this cause some problem in the Nigerian teaching hospital, because there are many outpatients that will be on queue at the clinic to compete for the limited appointments opportunity with physician (Arodiogbu, 2005). As a result of this, there is long waiting time before an NHIS outpatient can have access to see the Doctor or Physician. Thus access and appointment availability become the key component of outpatient satisfaction within the NHIS medical system.

Due to the major problem of long waiting time facing by many outpatient and most especially the NHIS outpatient in the Nigerian Teaching Hospitals (Thatcher, 2005). There is need to develop a web based NHIS outpatient medical appointment booking system to help patients to book appointment and access their medical records online. It aims to reduce the number of missed appointments, unnecessary outpatient queue at the clinics, and long waiting time of NHIS outpatients at the clinics. The system will enable the NHIS outpatients to login with their NHIS number to appointment booking interface and view the scheduling calendar for the available physicians, available time and room number of the available physicians. Then book an appointment by selecting from the list of available personnels that conform to the patient time. This paper presents a web based NHIS outpatient medical appointment booking system which 
creates a user friendly environment for patients and healthcare personnels to use. The aim is to empower the patient to book and manage their own appointments at their convenience, shortening the unnecessary waiting time for consultation.

\section{LITERATURE REVIEW}

\subsection{Waiting Time}

Waiting time simply means a period of time which one must wait in order for a specific action to occur, after that action is requested or mandated (Fernandes et al., 1994). Patients' waiting time has been defined as "the length of time from when the patient entered the outpatient clinic to the time the patient actually received his or her prescription" (Jamaiah, 2003). It is defined as the total time from registration until consultation with a doctor. There were two waiting times, the first is time taken to see a physician and the second is time to obtain medicine (Suriani, 2003). This paper deals with the waiting time to see physicians.

Long waiting times are a serious problem for patients using urban health centre's in developing countries (Bachmann, 1998). A block appointment system was introduced and evaluated in a large South African health centre. Waiting times of all patients were measured over one-week period before and after the implementation of appointments. Focus groups and individual interviews were conducted with staff and patients. After introducing appointments, patients with acute and chronic illnesses and having appointments had significantly shorter waits time than similar patients without appointments (Mahomed, 1998).

Appointments had no benefits for patients not seeing doctors or collecting repeat medication. There was, however, an overall increase in patients' waiting times after introducing the system, mainly due to one typical day in the follow-up study. Focus groups and interviews revealed that staff were skeptical at baseline but at follow-up were positive about the system. Patients were enthusiastic about the appointment system at all stages. The study shows that block appointments can reduce patients' waiting times for acute patients, but may not be suitable for all patients. Staff and patients had different views, which converged with experience of the new system (Mahomed, 1998).

\subsection{Patients' Appointment System}

A patient appointment system or appointment schedule for health care center started long time ago (Harper, 2003). Management of patients' appointments has earlier works and has developed simplified queuing models and fairly static scheduling conditions. Another attempt was made to calculate the waiting time between patient and doctor using the mathematical queuing models to minimize waiting time (Gamlin, 2003). However; traditionally the appointment system has considered that the doctor time is more important than patient time (Wijewickrama, 2005). So an appointment system was designed to minimize the doctor idle time but current designing of an appointment system is based on decisive factors with respect to both the patient and doctor (Takakuwa, 2005).

The patient appointment system has complex structures because it represents the patient appointment time in the healthcare center and controls the patient waiting time based on the type and the period of patient appointment (Gamlin, 2003). Moreover, a patient appointment system is 
meant for: managing doctor's time, reducing patient's waiting time, reducing doctor's idle time, reducing nurse's idle time, and improving the quality of service in the health care (Harper, 2003).

\subsubsection{Appointment Delay}

Past research shows that the longer the appointment delay which is defined as the time between the day a patient requests an appointment and her actual appointment date, the higher the chances that he/she will cancel or not show up (Gallucci et al. 2005). This suggests an obvious way of minimizing no-shows and cancellations: this is done by asking the patients to come right away or make appointment requests on the day they want to be seen (Murray, 2000). This is called an open access (OA) or advanced access policy (Tantau, 2000), and of late it has become a popular paradigm in practice and the subject of active research. Several authors report on their experiences in implementing OA, both positive and negative (Dixon et al. 2006). Some practitioners strongly advocate OA (Murray and Tantau 2000), and there are some who are strongly against it (Lamb, 2002).

\subsubsection{Managing Patients' Appointment system}

According to Dexter (1999), managing patient appointment system is a computer application used to manage and reduce the patient waiting time in the health care center. Some health care centers do not use any appointment system. So it has a longer average patients' waiting time than the health care center that adopts the patients' appointment system. While patients can wait for more than one hour to be attended to by a physician in a health care center, they also can feel that they are being disregarded and treated unfairly. So when patients are given the time of appointment in a health care centre, they can evaluate the quality of service in the centre (Dexter, 1999).

Hence, developing patients' appointment process for health care centres necessitates the use of a sophisticated queuing model that captures much of the real system's features (saving time, reducing idle time, etc). Therefore the appointment schedule represents the real situation in the health care centre faced by patient appointment schedulers (Rohleder, 2002). On the other hand, the standard practice for scheduling and processing patient appointments are based on the nature of treatments of the patients and that better approaches more sensitive to patient needs are desirable (Klassen, 2002).

\subsection{Online Booking System}

An online system is also known as a web based system. A web is made up of page that is commonly known as web page or web site, and a web site is a computer program that runs a web server that provides access to a group of related web pages (Alex, 2000). A system is a set of independent components working together to achieve a common objective. Therefore a web based system is a system that is accessible over the internet by a user in order to achieve a particular task for a given purpose. The Internet is a system that is use to connect computers and computer networks. It helps to link millions of computer networks all over the world and it allows the users to get information stored on other computers from a long distance (James, 1999).

According to Chua (2010) the public demand for better healthcare system and the alarming number of missed appointments have forced the healthcare sector to recognize how they deliver care services. With the advance of IT technology today and seen healthcare system as a critical 
system, appointment booking system lies at the intersection of delivering efficient, dependable and timely access to health services. The conventional way of appointment booking is via fax, phone or email. But with the growing internet penetration, healthcare industry is moving towards the use of an online appointment booking system.

A web-based appointment system is used in Taiwan; everyone is required to enroll in the national health insurance program. When one needs health service, he shows his health insurance card to doctors in an hospital to start with. There are several ways of making an appointment. A person can either go to the hospital directly for consultation day by day or make an appointment from home through phone call or email if his condition is not emergent (Gruca, 2004). The Internet provides a wide range of technologies that enable hospitals to communicate with their patients. Recently, as the prevalence of Internet increasing, many hospitals initiated the website appointment system. Electronic patient-provider communication promises to improve efficiency and effectiveness of clinical care (Wakefield, 2004).

\subsection{Existing Hospital Appointment Schemes}

One application developed to manage patients' appointment scheduling has used exponential enter arrival times. This model assumes that the exponential enter arrival times could not be directly validated by date, and it is limited due to the nature of the appointment scheduling (Rohleder, 2002). Since appointments are scheduled in the future, the exact model of call arrivals will only have limited impact on measures related to the time between the call and the appointment time. For this reason, the challenge for making appointment system is designing a suitable system based on the health care procedure environment (Klassen, 2002). Hence, the appointment provider in the health care center can schedule a patient into an appropriate time slot on a given day.

Klassen (2004) developed another method for managing patients' appointment using multiple schedule appointment in multiple period environments. Patients can call for any appointment time but if the period time is full, they should replace the appointment to another time. Moreover, various combinations for multi appointment and double booking are measured and recommended for different operational use depending on the health care environment because the varying appointment request has little effect on appointment system performance, especially maintaining acceptable performance, except when the system has the overloaded option (Rohleder, 2004).

Many studies about patients' appointment have found that there are rules or policies for scheduling appointment system such as no scheduling for more than 20 or 30 clients and the best schedule is to place two patients in the first appointment and spread the rest consistently over a period based on average service times (Klassen, 2004). On the other hand, a patient can call for an appointment without knowledge of the type of appointment and appointment queue number and the patient is not aware whether the appointment is variable or not. Sometimes the exact duration for each patient can be known but at other times this is unknown (Rohleder, 2004).

Another system developed by Mustafa, (2004) allows a registered patient, having user name and password, to access and explore the list of physicians alphabetically and select a physician whose email contact and profile are also provided. A patient can also view the physician working calendar to find out his/her working and non working day to make an appointment. When the patient selects, view calendar the patient can then choose any valid day in any month to make an 
appointment (Mustafa, 2004). After that, the patient will receive an e-mail from the system to confirm the appointment time or to inform the patient that the selected time is already taken by another patient or blocked by the physician. In general, the patient appointment system provides all the choices and the capabilities to the patients, such as selecting a physician, selecting the time of appointment, and allows them to access the health care system day or night and schedule their own appointments using the Internet without spending time holding for a nurse or having lengthy phone calls.

Wijewickrama and Takakuwa (2005) opine that the health care operating time (due time) is from 8:30 am to 5:30 pm during the week days. Throughout this period, four types of patients arrive to have a consultation appointment in the health care center-appointed patients, same day appointment patients (walk-ins), patients who come for a medical test and new patients (Wijewickrama, 2005). Patients who have appointments are given priority over those who walkin for consultation. Consequently, these latter patients have to wait a long time in the waiting room to meet a doctor even if the consultation time only last few minutes (Takakuwa, 2005).

Porta-Sales et al. (2005) developed another system. The main concept of the system is contacting, screening and scheduling appointment with the health care center initially by an expert nurse and the patient initiating contacting with the health care center using the telephone. Moreover, the health care center can be accessible from different places. So there should be PC resources and PC consultations to be accessed from different sources, from other hospitals, from general practitioners, or even from the patients themselves. Porta-Sales et al. (2005) studied 534 patients for a period exceeding one year. After the first visit, 195 patients did not return for the second scheduled appointment and 203 patients had progressed on to the third scheduled visit. The main reason given for the scheduled visits was admission into the health care; the median time-lapse between the first and second visit was 21 days, between the second and third was 27.5 days and between the first and third was 48 days. Comparing patients, who did not attend the three consecutive visits with those who did, indicated that the former had (at the first visit) a lower performance status.

Su et al. (2003) studied in a private hospital which has several clinics. For each clinic, the average patient load is 20 per consultation section (morning or afternoon) and the health care system adopts both a patient appointment model and patient registration model. The system allows patients to have self-selected specific physicians for consultation and registration (Shih, 2003). The management appointment system studied by Su and Shih, (2003) is based on the first 20 reserved for scheduled patients, after that, only seven are offered for scheduling. Odd numbers after 20 are left for walk-ins. The arrival time of the first patient is assumed to be the same as the clinic starting time. The scheduled patients are assigned based on 3-main intervals and are also informed about their appointed arrival times (Su, 2003). If the scheduled patient does not appear on time, the next available patient receives consultation immediately. The management operating philosophy of services here is based on "first in, first seen" to limit patient waiting time. Therefore, patients can walk-in to see a physician, when patient shows up at the appointed time (Shih, 2003).

Some of the existing appointment booking system have some limitations and the system developed in this research eradicate the limitations of the existing system in confirming patient medical appointment by sending an email to the patient if the appointment have being confirmed or not. It will also enable the patients to view and monitor their medical records online. 


\section{METHOD}

In order to achieve the objectives of this research, the researchers personally went to three Nigerian teaching hospitals within south western Nigeria with focus on NHIS patients and observed the appointment schedule in those hospitals. Also as a follow up, the researchers interviewed 4 physicians and 10 patients in each of the teaching hospitals in order to assess the manual appointment and booking method. In addition, data on appointment booking and schedule were collected through structured and unstructured personal interview. Some of the forms used in the outpatient clinics on appointment booking were also collected and were used as a guide in order to develop the online medical appointment booking system for NHIS patients in Nigeria.

Database was developed using MySQL for the system. In the process of developing medical appointment booking system for NHIS patients, records were stored in the database. MySQL in wampserver was used to create and populate the database. The medical appointment booking system for NHIS patients in Nigeria was implemented using dream weaver, and PHP. Apache was used as the server to provide basic functionality of the web GIS. PHP was used as a scripting language to program the server side that manipulates the knowledge in the database.

\section{SYSTEM ARCHITECTURE AND USE CASE DIAGRAM}

\subsection{System Architecture}

The architecture is structured to allow users to make use of portable computer system, desktop computer system, and mobile phone as web browser to access the booking system. Client-server architecture was used and we used thin client-server. The medical appointment booking system has two components namely: the server-side and client-side that run on the browser.

In the client approach almost all the processing work was done on demand at the server end and the client task was to display data and information on the screen. While in thin client-server architecture, the web browser is the client. This architecture was used because with it users will not be required to install any software on their PCs expect a standard web browser, which often come, with most PC operating system and almost all the current standard mobile phone.

Clients would also not require any powerful PC; users can use any PC with a web browser such as laptop/notebook, mobile phone, and desktop PC. The servers would require higher configuration (in terms of hardware) because it would be regularly subjected to heavy load. Figure 1 depicts the medical appointment booking system architecture.

\subsection{Use Case Diagram}

The use case diagram is used in presenting the system requirements of any proposed system. A use case is a realistic description of the workflow of the system and it is used to explicitly describe the intentions and actions of users. The use case diagram, which present the system requirements are used to show how the proposed system work in practice. The interaction between actor and use cases are also described using use case diagram. The use case diagram of the medical appointment booking system is presented in figure 2 . 
International Journal of Computer Science \& Information Technology (IJCSIT) Vol 6, No 4, August 2014

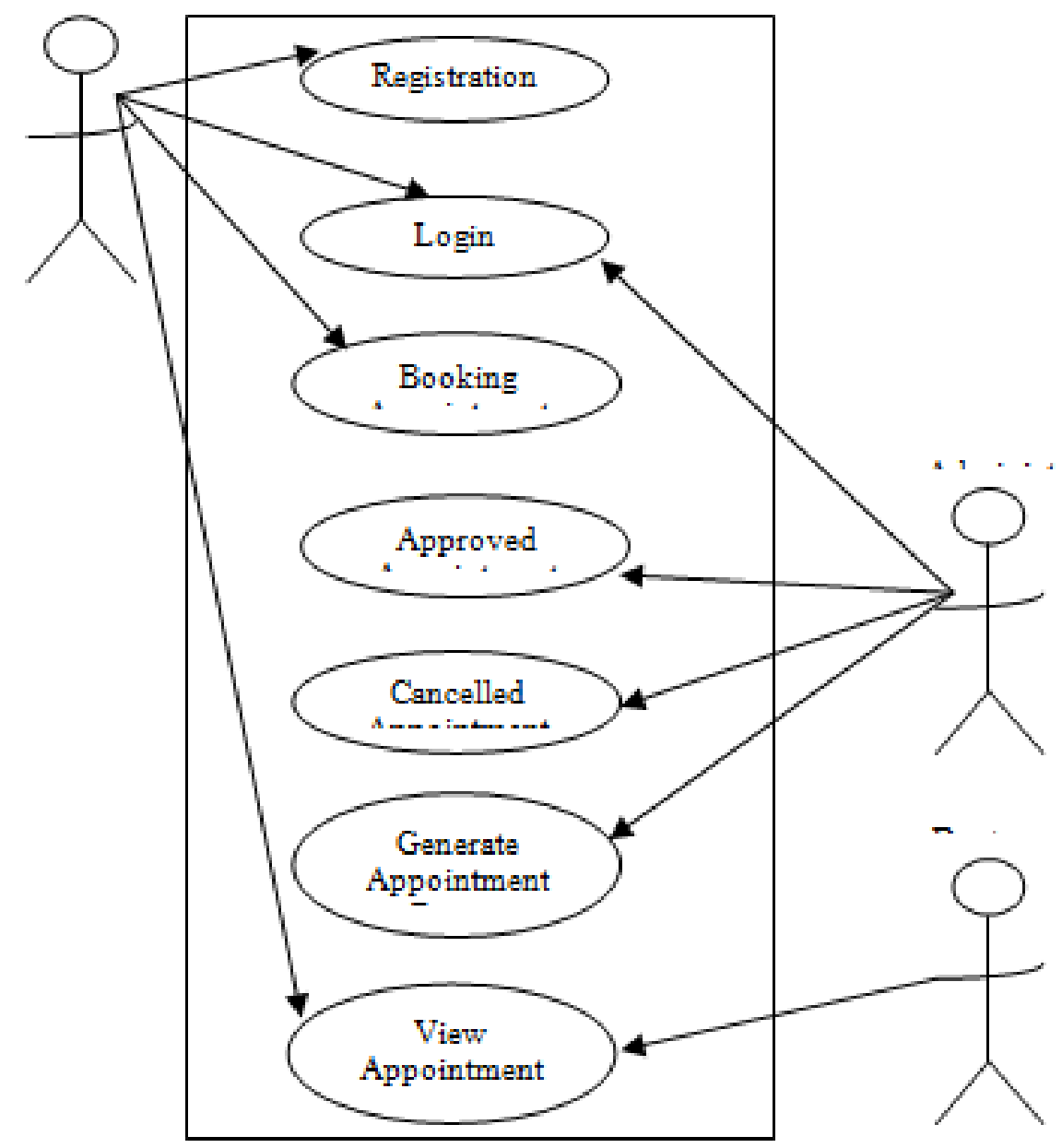

Figure 1: Medical Appointment Booking System architecture 


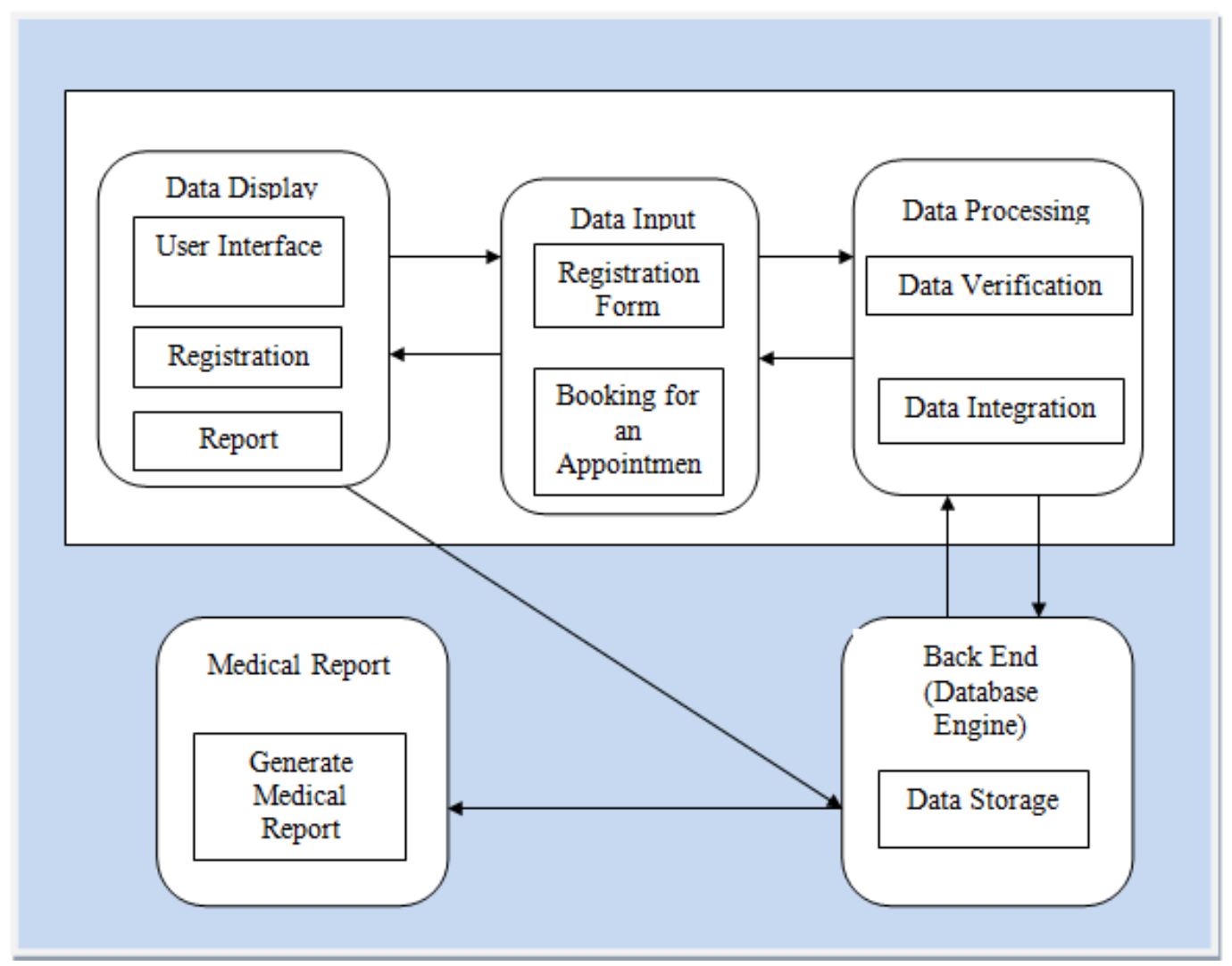

Figure 2: Web Based Medical Appointment Booking System

\section{THE ONLINE MEDICAL APPOINTMENT BOOKING SYSTEM}

The user interface allows the user to navigate the system and communicate with the database. The system interface design is simple enough to be the use for anyone with little knowledge of computer operation and the system is user friendly and made easy to use through the use of Windows, Interface Menu and Pointing devices (WIMP) which is very important in computer graphics design. The inputs to the online medical appointment booking system are captured through the user interfaces available in administrator's web account and the individual patient's web account. A login Menu, which contains a user-friendly interface for capturing valid registration code, username and password, is available for each patient to logging in to their various accounts. In each patient account, data pertain to the patient and available doctor are entered and appointment booked are submitted. All these captured data are stored in the database.

\subsection{Patient Registration Menu}

There is need for every new patient to register before they can access the system, a patient must have a username and password which they will use to login before they can book for medical appointment. Any patient that does not register will not be allowed to access appointment booking Menu because to login to the appointment Menu requires a username and a password. Figure 3 shows the patient registration Menu and after the patient has successfully registered as a 
new user to use the system. This is the Menu that will be display as a confirmation Menu for the registration and this Menu can be print out by the patient and this is depicted in figure 4 .

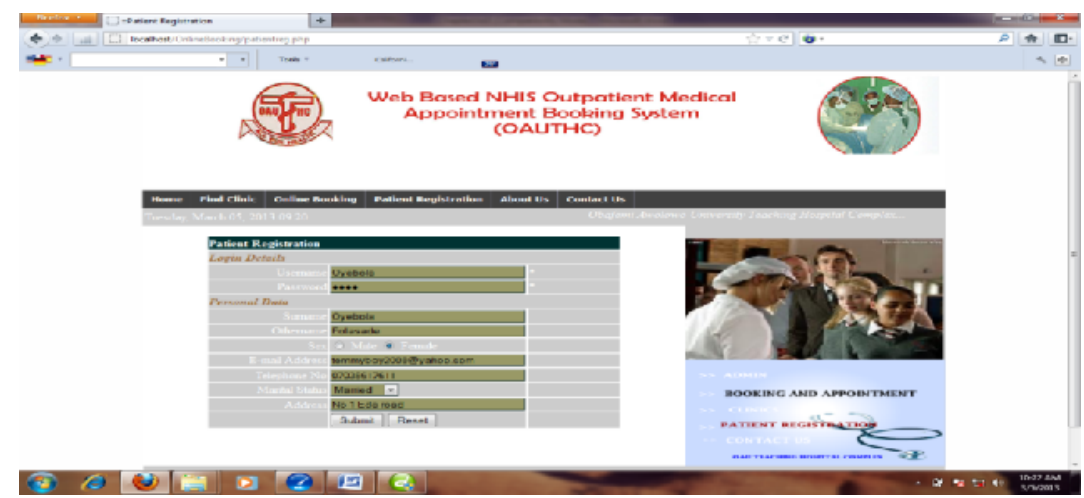

Figure 3: Patient Registration Menu

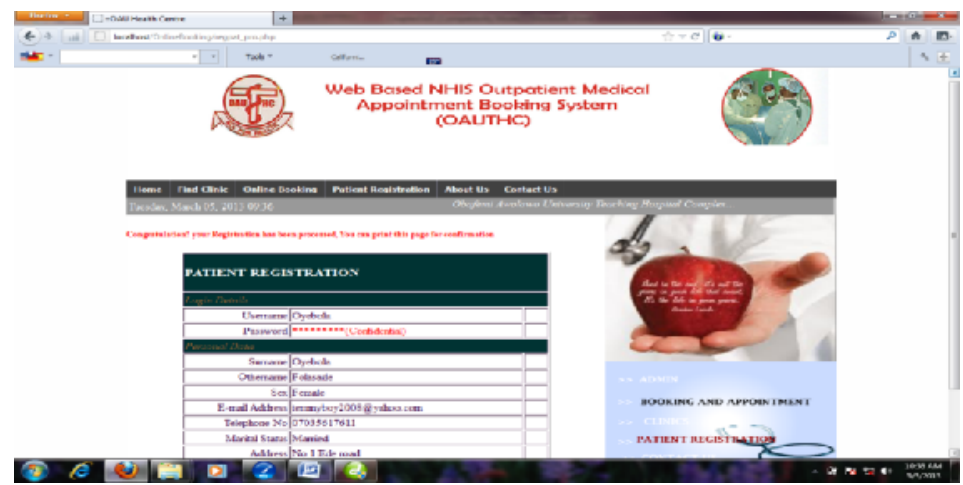

Figure 4: Output of Patient Registration

\subsection{Patient Activity Menu}

The patient activity Menu which is depicted in figure 5 displays the list of link of operation that a patient can perform with the system; the following are the activities a patient can perform.

- Appointment Booking

- View Appointment Report

- Log Out 
International Journal of Computer Science \& Information Technology (IJCSIT) Vol 6, No 4, August 2014

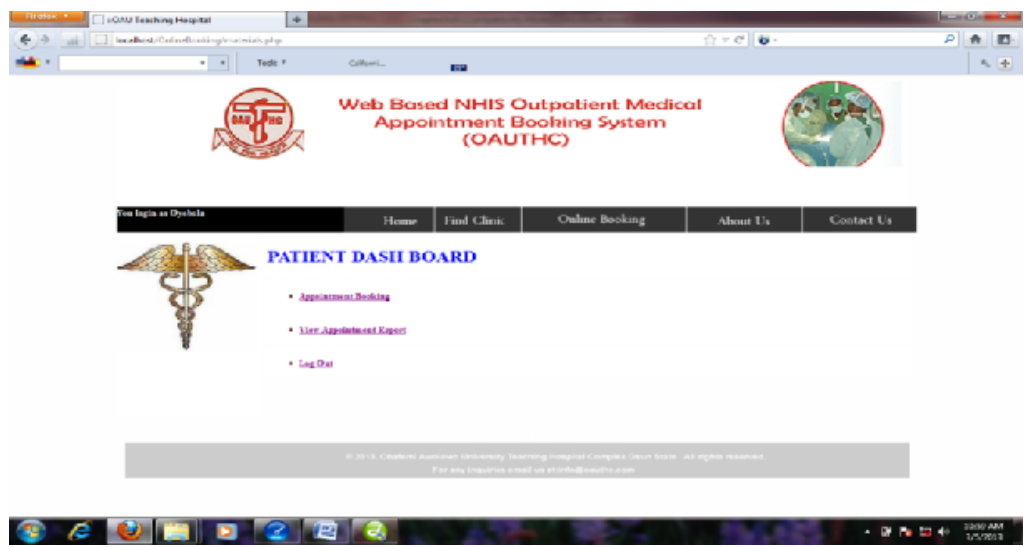

Figure 5: Patient Activity Menu

\subsection{Weekly Calendar}

This enables the patients to book for medical appointment by clicking on the link and the above Menu will be display. This Menu enables the patient to view the calendar as scheduled by the system administrator based on the weekly activities of the doctors at the Obafemi Awolowo University Teaching Hospital complex. When a patient view this calendar then he/she will know the doctor that is available at a particular time and make his/her appointment based on the schedule. After viewing the calendar, the patient can then clink on next button to continue with the appointment and this is shown in figure 6.

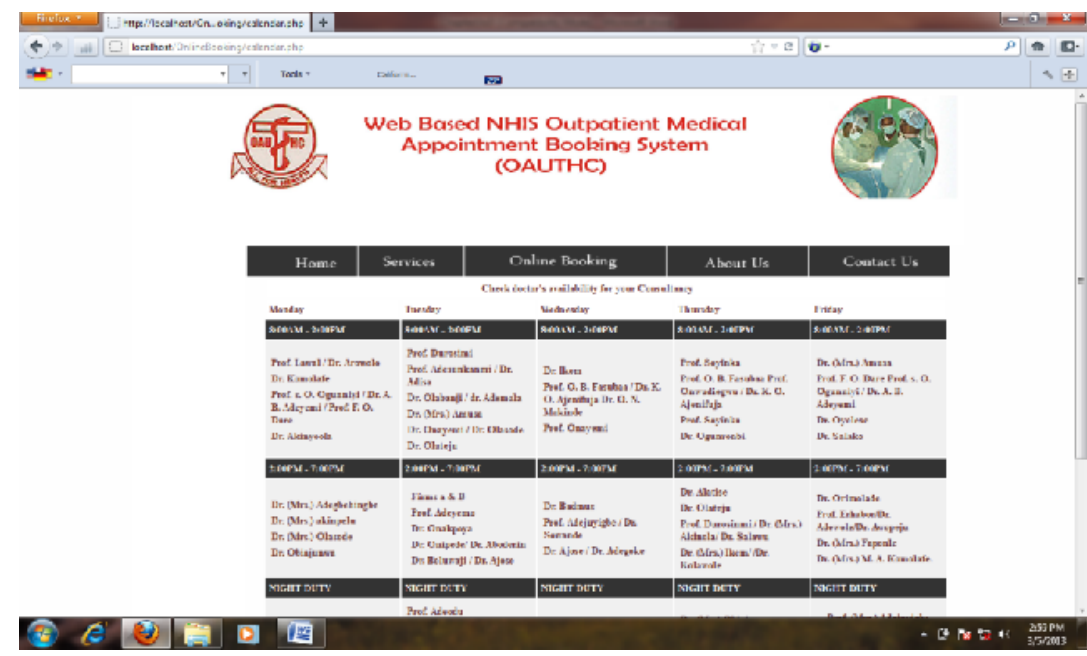

Figure 6: Weekly Calendar

\subsection{Patient Appointment Menu}

The patient appointment Menu depicted in figure 7 is to allow the patient to book for medical appointment based on the calendar already viewed. All the fields provided must be entered and then clink on submit button, the output Menu will be display. The appointment output Menu depicted in figure 8 shows the confirmation of the appointment already booked by a patient with a 
doctor. The appointment will then be treated by the administrator for final confirmation, the appointment can then be approved or disapproved based on the doctor availability. The patient can also print as a confirmation for their appointment. The patient can then view the appointment status weither the appointment has been approved or not. The patient can clink on the link name to view appointment report and the following Menu will be display.

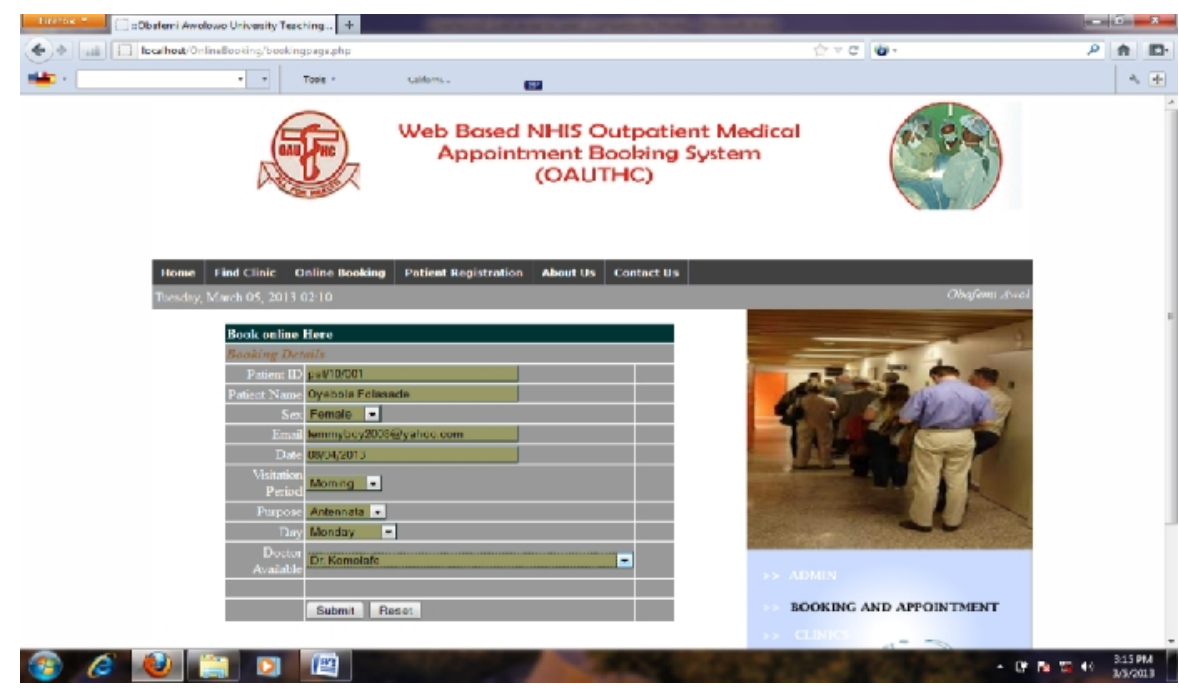

Figure 7: Patient Appointment Menu

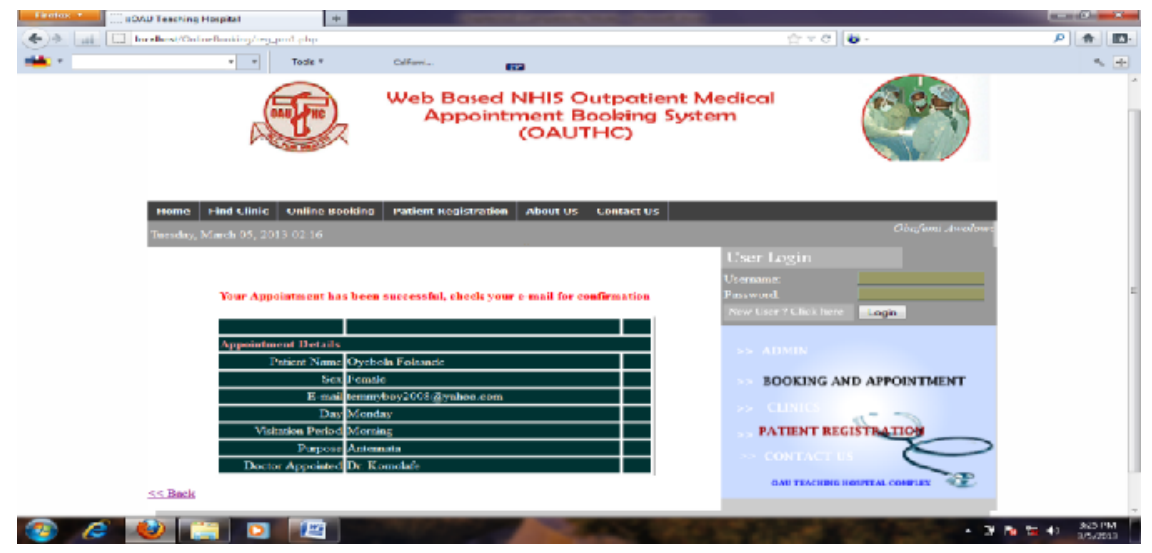

Figure 8: Appointment Output Menu

\subsection{Patient Report Menu}

The patient report Menu depicted in figure 9 allows the patient to view their medical report by selecting their patient ID and then clink on the search button in other to view their appointment status from their account as alternative means of viewing their appointment status instead of going to their emails. This status can as well be view from their email but in other to save time, the patient status can be view directly from the user account. When the search button is clink, then the output Menu will be display. 
The menu shows the patient appointment report where the patient can view the status of their appointment. If their appointments have been confirmed by the system administrator from the admin Menu, the status column will indicate it and a shot message will be displayed at message column. The Menu depicted in figure 10 can be printed out by the patient.

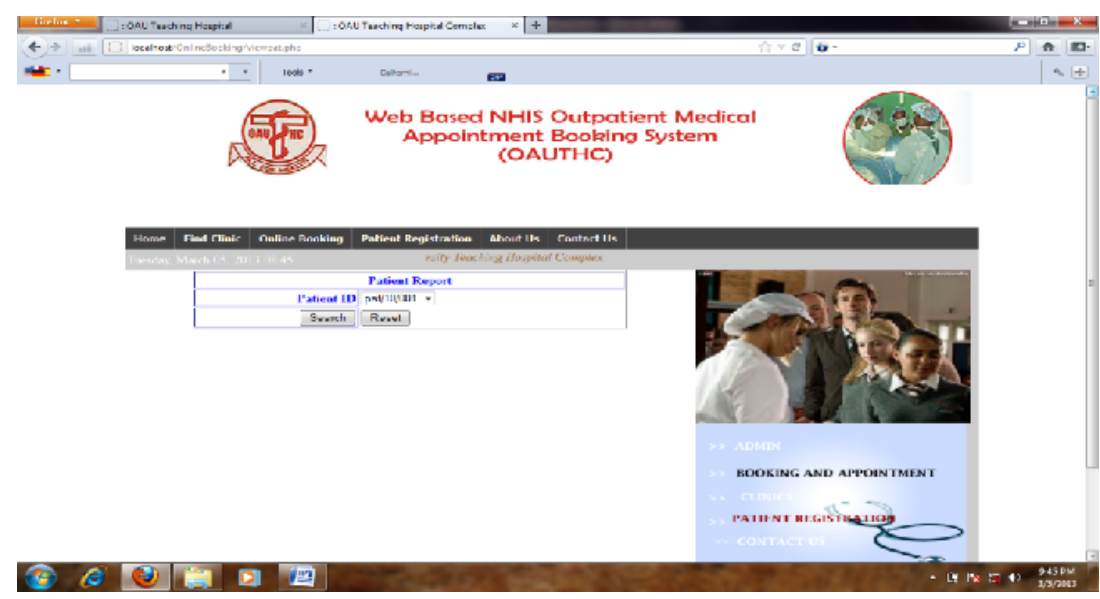

Figure 9: Patient Report Menu

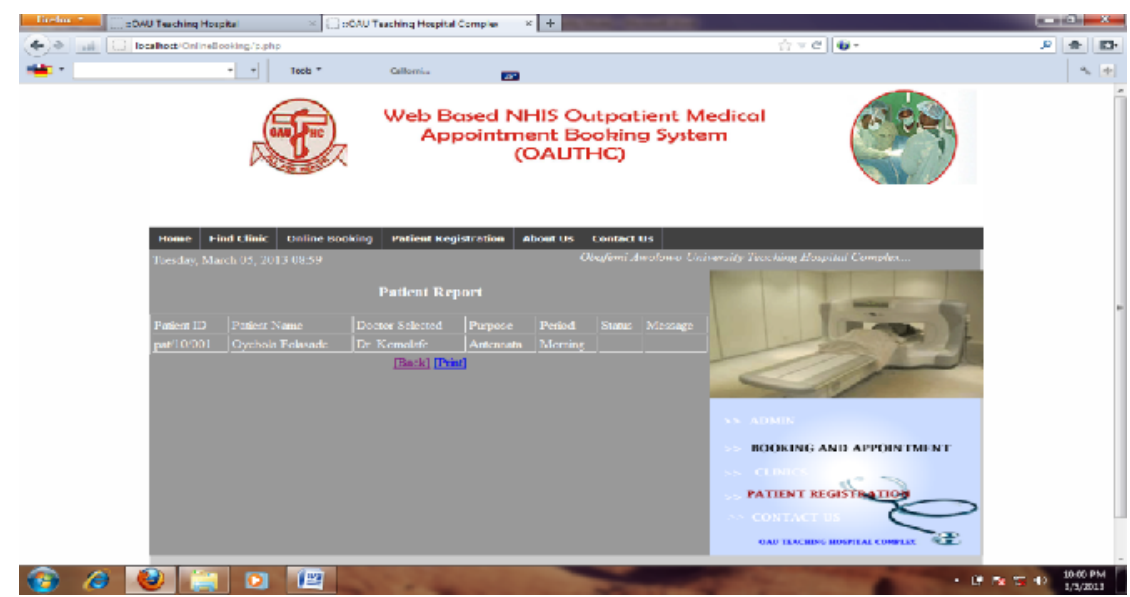

Figure 10: Patient Appointment Report Menu

\subsection{Administrator login Menu}

The administrator interface allows the administrator to be able to implement editing, inserting, deleting and to check the overall result of all registered outpatients. An administrator could be a doctor or nurse who is able to schedule appointment, generate appointment report and view the appointment report of patients that has registered all the necessary information needed. The patient has a patient identification number in order to search for the information stored in the database of the system. The information available will be displayed to the user by the system if he had entered valid registration number. Figure 11 shows the administration login Menu. When an administrator login the following menu displays.

This Administrator activity menu represented in figure 12 shows the activities that the administrator can perform and the following activities can be performed 
- Check Appointment (Approved or Disapproved)

- Cancelled Appointment

- Prepared Doctor's Roaster

- Generate Appointment Report

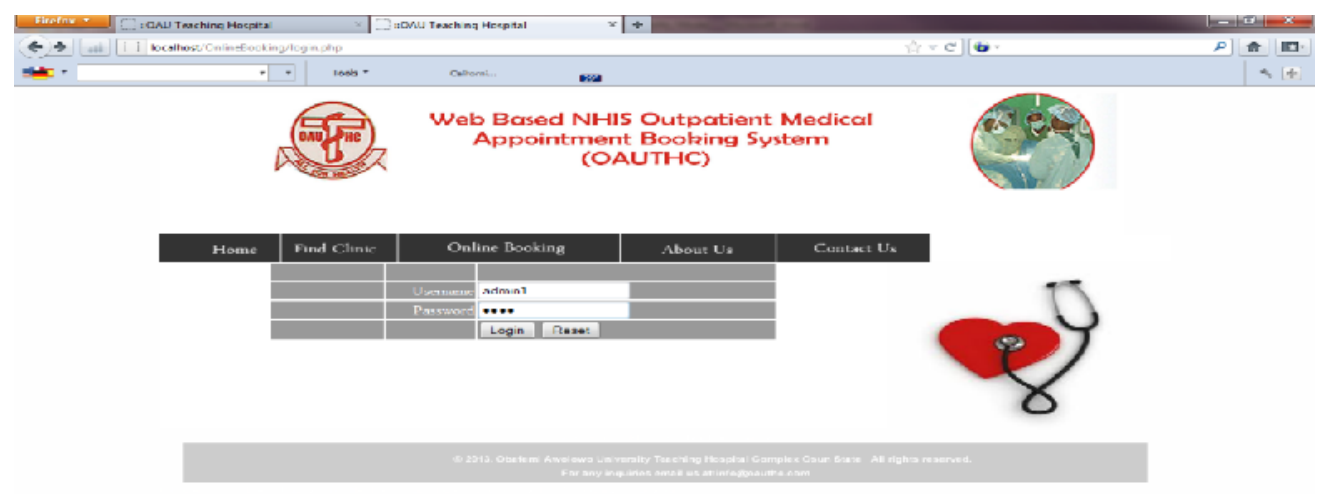

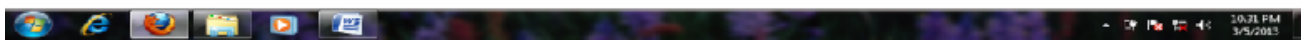

Figure 11: Administrator Menu

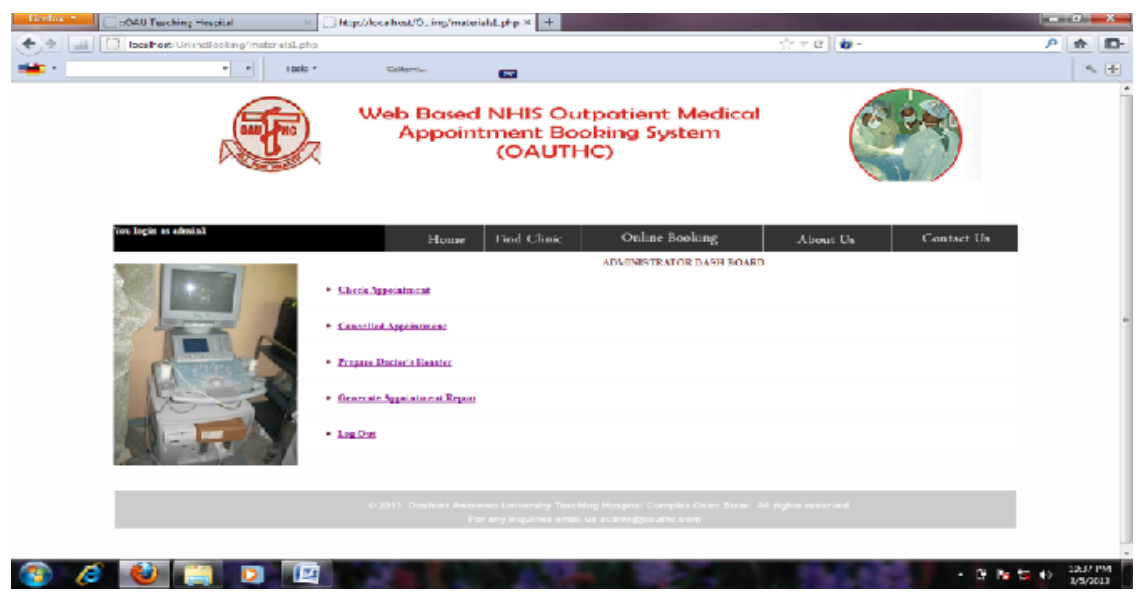

Figure 12: Administrator Activity Menu

\section{CONCLUSION}

With the development of web based NHIS medical appointment booking system, patients are able to book and manage their own appointment with ease. They will be reminded of their appointments via SMS/email that will be promptly sent to them before their appointment date. The system itself also provides a quick view of their appointment at the Home page. These functions could indirectly help to reduce the number of missed medical appointments and patients no-show up for their appointment. Patients would be notified via SMS/email if their appointment were affected, when there is urgent needs of the service provider at other place or in case of any situation that can result to the absence of the service provider. 
Patients could also track and monitor their own appointment record with this system. However, the display of bio-data such as X-rays and laboratory results are not included in the system due to technical constrain. The system will not be able to diagnose or prescribe drug for usage. The system is mainly designed to facilitate appointment booking between the patient and the health personnel. In compensation, additional modules such as Announcement, Medical case record and block/unblock schedule will further enhance the usability and functionality of the system and allow a flexible management of patients appointment.

The system delegates some administrative work to the patients by allowing them to manage their own appointment and personal profiles. Time will not be wasted on converting paper-based appointment record into electronic-based. The system further helps to reduces healthcare personnel workload by allowing them to generate medical reports easily. They could now maximize their competence and allocate more time to maximize service quality.

\section{REFERENCES}

1. Adesina, D. (2009). The National Health Insurance Scheme. Retrieved October 3, from http://www.nigeriandoctor.Com/news.php?extend.85D.

2. Arodiogbu I.L. (2005). Introducing Social Health Insurance to solve problems of poor health Sector in Nigeria. MA Thesis. University of Leeds.

3. Alex Chaffee (2000-08-17). "What is a web application (or "webapp")?". Retrieved 2008-07-27.

4. Bailey, N. (1952). A study of queues and appointment systems in hospital outpatient departments, with special reference to waiting times. Journal of the Royal Statistical Society, A14, 185-199.

5. Bailey, N. (1954). Queuing for medical care. Applied Statistics 3, 137-145.

6. Bailey NTJ. A study of queues and appointment systems in hospital out-patient departments, with special reference to waiting times. J Royal Stat Soc 1952;14:185-99.

7. Cayirli, T, E. Veral, and H. Rosen. (2004). Assessment of patient classification in appointment systems. 1st Conference of the POMS College of Service Operations, New York, NY, USA.

8. Cayirli, T, E. Veral, and H. Rosen. (2006). Designing appointment scheduling systems for ambulatory care services. Health Care Management Science 9, 47-58.

9. Denton, B., and D. Gupta. 2003. Sequential bounding approach for optimal appointment scheduling, IIE Transactions, 35, 1003-1016.

10. Fernandes C, Daya M, Barry S, Palmer N (1994). Emergency department Patients who leave without seeing a Physician: The Toronto Hospital experience. Ann. Emer. Med., 24: 1092-1096.

11. James Duncan Davidson, Danny Coward (1999-12-17). Java Servlet Specification ("Specification") Version: 2.2 Final Release. Sun Microsystems. pp. 43-46. Retrieved 2008-07-27.

12. Johnson, J. A. \& Stoskopt, C. (2009). Comparative Health Systems: Global Perspectives.

13. Kannegiesser, L. (2009). Nation Health Insurance Scheme to Boost Generics Market in Nigeria.

14. Klassen, K. J., T. R. Rohleder. 2004. Outpatient appointment scheduling with urgent clients in a dynamic, multi-period environment. Internat. J. Service Indust. Management 15(2) 167-186.

15. Koole, G., and G. Kaandorp. 2006. Optimal outpatient appointment scheduling, Working Paper, Department of Mathematics, Vrije Universiteit Amsterdam, The Netherlands, http://www.math.vu.nl/ koole/articles/hcms07b/ (checked on May 24, 2007).

16. NHIS (2009). NHIS - News: States Partners with NHIS. Online available at: http://www.nhis.gov.ng/index.php? Retried October, 3, 2009.

17. Oba, J. O. (2008). Nigeria: Yar'Adua and the Resuscitation of Health Sector. Online available at: http://allatrica.com/stories/200806021431.html. Retrieved October 3, 2009.

18. Thatcher TD (2005). Outpatient waiting time in Jos University Teaching Hospital (JUTH). Highland. Med. Res. J., 3(1): 36-42. 